\title{
Aircraft Combat Survivability Calculation Based on Combination Weighting and Multiattribute Intelligent Grey Target Decision Model
}

\author{
Lintong Jia, Zhongxiang Tong, Chaozhe Wang, and Shenbo Li \\ Aeronautics and Astronautics Engineering Institute, Air Force Engineering University, Xian 710037, China \\ Correspondence should be addressed to Lintong Jia; jialintong406@163.com
}

Received 20 September 2015; Revised 22 December 2015; Accepted 13 January 2016

Academic Editor: Danielle Morais

Copyright (C) 2016 Lintong Jia et al. This is an open access article distributed under the Creative Commons Attribution License, which permits unrestricted use, distribution, and reproduction in any medium, provided the original work is properly cited.

\begin{abstract}
Aircraft combat survivability is defined as the capability of an aircraft to avoid or withstand a man-made hostile environment, which has been increasingly important. In order to give a rational calculation of aircraft combat survivability, an integrated method based on combination weighting and multiattribute intelligent grey target decision model is proposed. Firstly, an evaluation index system containing susceptibility index and vulnerability index as well as their subindexes is established. Then a multiattribute intelligent grey target decision model is introduced. A combination weighting method is brought up based on a modified AHP (analytic hierarchy process) method and the entropy method, offering a rational weight for various indexes. Finally, utilize the multiattribute intelligent grey target decision model to assess the aircraft combat survivability of aircraft, verified by a practical case of five aircraft. The results show that the proposed method is effective and has a great value in engineering application, which will provide useful references for other projects' evaluation.
\end{abstract}

\section{Introduction}

Survivability is defined as the capability of a system, including its crew, to avoid or withstand a hostile environment without suffering an abortive impairment of its ability to accomplish its designated mission [1]. For the aircraft combat survivability, Robert E. Ball defined it as the capability of an aircraft to avoid or withstand a man-made hostile environment. With the development of precision guided weapons, particularly radar-guided missiles and infrared-guided missiles, aircraft combat survivability is becoming increasingly important. The American Department of Defense has taken aircraft combat survivability as a basic design criterion. For example, the Navy MIL-HDBK-2069-1997 aircraft survivability stipulates that the survivability criterion should be carried out throughout the cycle life. The newest combat aircraft, F/A-18E/F Super Hornets, F/A-22 Raptor, and F-35 Lighting II, to name a few, has adopted survivability strengthening measures from the initial research phases.

Usually survivability can be subdivided into susceptibility and vulnerability, referring to the inability of an aircraft to avoid and withstand the man-made hostile environment, respectively. Aircraft combat survivability can be also defined as the probabilistic values that the aircraft would survive in man-made hostile environment, with the antithesis killability. The more susceptible and vulnerable the aircraft in the hostile environment, the more killable and lower survivable the aircraft.

Wang et al. construct an analytic model for aircraft survivability assessment based on the theory of stochastic duel considering the encounter process [2]. Konokman et al. carry out the aircraft survivability analysis considering vulnerability against fragmenting warhead threat [3]. Li et al. propose a vulnerability modeling and computation method based on product structure and CATIA and assess the effects of redundant technology [4]. Erlandsson and Niklasson argue a five-state survivability model, including undetected state, detected state, tracked state, engaged state, and hit state [5]. Shi et al. build an aircraft antagonistic model and a warfare model based on the agent theory [6]. These simulation methods bring a great deal of calculation and complex procedure, 


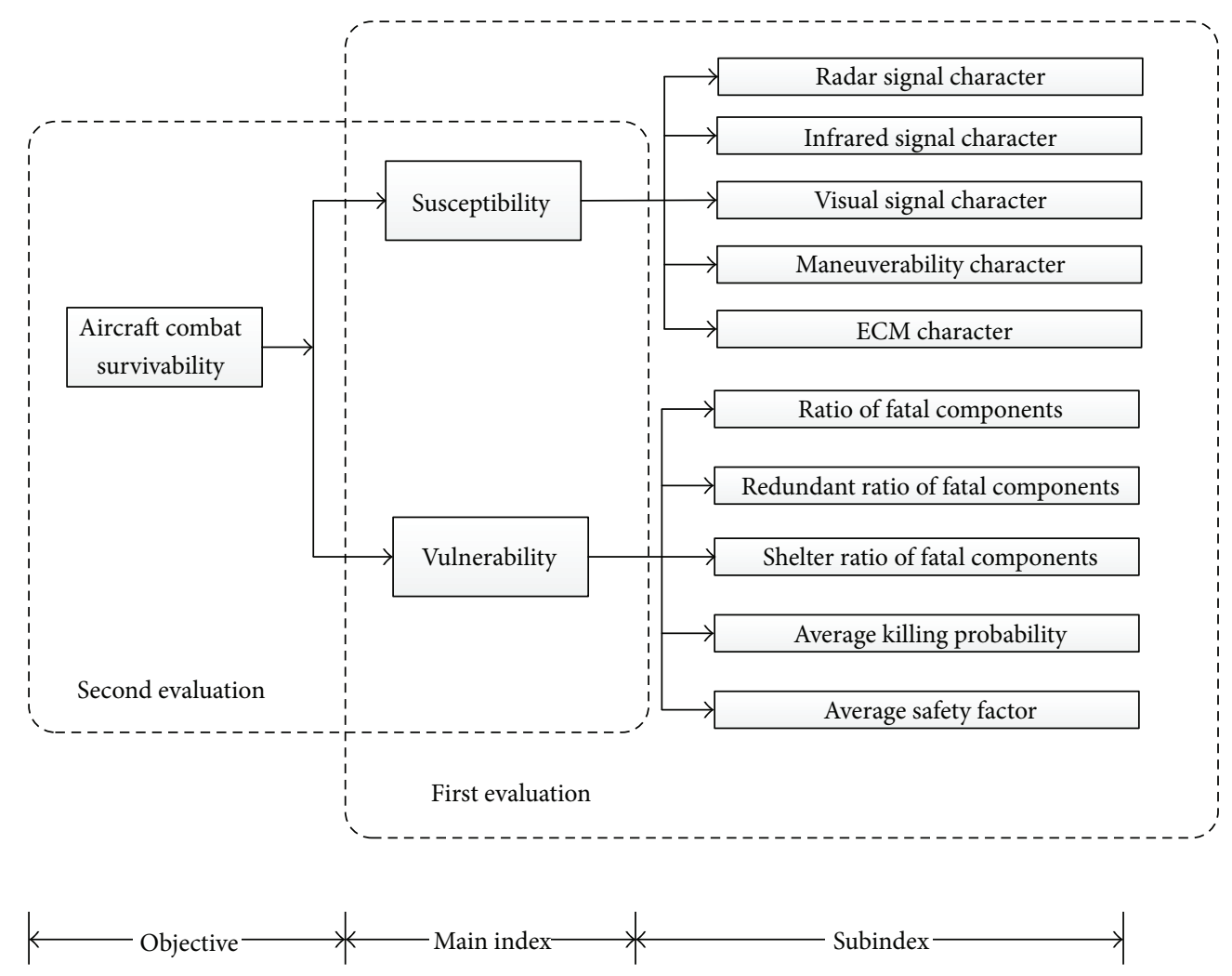

FIgURE 1: Aircraft combat survivability evaluation index system.

which impose restrictions on the application especially in the initial research without accurate data.

Multicriteria decision method is a useful replacement through ranking and selecting a finite number of alternative plans [7]. Grey target theory is the application of nonuniqueness principle in decision theory, which has been used in many good fields. A new multiattribute intelligent grey target decision model is introduced into the aircraft combat survivability analysis. In this paper, firstly, the aircraft combat survivability evaluation index system is established containing susceptibility index and vulnerability index. Secondly, a multiattribute intelligent grey target decision model is established based on the index system. Then, a combination weighting method is brought up based on a modified AHP (analytic hierarchy process) method and the entropy method, offering the combination weight to the multiattribute intelligent grey target decision model. In the end, a numerical example containing five aircraft is given, proving that this method is practicable and effective.

\section{Aircraft Combat Survivability Evaluation Index System}

Survivability can be subdivided into susceptibility and vulnerability. So the index system contains susceptibility index and vulnerability index. The index is established in hierarchy. The main index is susceptibility index and vulnerability index. The subindexes of susceptibility contain radar signal character, infrared signal character, visual signal character, maneuverability character, and ECM (electronic countermeasure) character, while the subindexes of vulnerability contain ratio of fatal components, redundant ratio of fatal components, shelter ratio of fatal components, average killing probability, and average safety factor, as is shown in Figure 1. The details are as follows.

2.1. Aircraft Susceptibility Subindexes. Considering the character of the aircraft, radar signal character, infrared signal character, visual signal character, maneuverability character, and ECM character are brought out.

Radar Signal Character. Radar signal is the most important signal character of the aircraft for locating, identifying, and tracking. Radar is one of the most lethal threats for aircraft. For example, early warning radars can provide airborne target information out of hundreds of miles, while ground control interceptor can even provide an accurate target location. From a simplified form of radar range equation in (1), we can see that RCS (radar cross section) is the one and only controllable parameter for the aircraft:

$$
\begin{aligned}
& R_{\max }^{4} \\
& =\frac{\left(\frac{P_{r} C_{t}^{2} \lambda^{2}}{(4 \pi)^{2}} \cdot \frac{1}{S_{\min } L}\right)(\sigma)}{} \quad F_{1}^{2} F_{2}^{2} \\
& \quad \text { Radar Capability } \text { RCS Atmospheric propagation }
\end{aligned}
$$


where $R_{\max }$ is the maximum range at which an aircraft can be detected, $C_{t}$ is the characteristic parameter of radar which varies for different type, and $F_{1}$ and $F_{2}$ are the propagation loss of signal emission and return.

So, we choose radar cross section as the evaluation index denoted by the sign $S_{11}$ with the unit square meter $\mathrm{m}^{2}$.

Infrared Signal Character. Infrared signal is another important signal character of the aircraft. With the development of stealth and antistealth techniques of radars, the aircraft's RCS has decreased significantly. However, compared with the environment, infrared signal is still significant even for the stealth aircraft. In the war area for 20 years, about $90 \%$ of airplanes were damaged by the infrared-guided missile [8]. Nowadays, IRST (infrared search and track) system, FLIR (forward looking infrared) system, and infrared-guided missiles have been significant threats by accurately locating aircraft. For point source infrared detector, the infrared range equation under uniform background can be written as $R \propto$ $\sqrt{I}$, where $R$ is the range at which an aircraft can be detected and $I$ is the aircraft's infrared radiation intensity. For most aircraft, the engines are the largest sources of thermal energy. So, we choose turbine inlet temperature as the evaluation index denoted by the sign $S_{12}$ with the unit Kelvin K.

Visual Signal Character. Visual signal is another important factor in determining overall aircraft detectability [9]. Air combat in visual range is still essential and visual acquisition before launch may be required. Contrails, engine exhaust glow, cockpit lighting, and luminescence may provide visual cues. Here, we choose size factor, which is closely related to the probability of visual detection as the evaluation index denoted by the sign $S_{13}$ with the unit meter $\mathrm{m}$. Size factor is defined as

$$
S_{13}=\sqrt[3]{\frac{3 * L * H * W}{4 * \pi}},
$$

where $L$ is the length of aircraft, $H$ is the height of aircraft, and $W$ is the wingspan of aircraft.

Maneuverability Character. Maneuverability is an effective means of defense for the aircraft against detection and attack. Supersonic maneuver is now the standard of new generation aircraft. Supersonic maneuver gives an aircraft a lower susceptibility and a higher survivability. Maneuverability character can be defined with the maximum allowable overload $n_{y \text { max }}$, the maximum steady turn overload $n_{\text {cir }}$, and specific excess power SEP as is shown in the following:

$$
S_{14}=n_{y \max }+n_{\text {cir }}+\operatorname{SEP} \times \frac{9}{300} .
$$

Electronic Countermeasure Character. Electronic countermeasure plays an important role in modern military affairs, including active and passive jamming, which is an effective mean to decrease aircraft susceptibility and enhance aircraft survivability. Electronic countermeasure equipment contains omnidirectional radar warning equipment, radar chaff dispensing device, infrared jammer, and infrared-guided
TABLE 1: Electronic countermeasure subindexes of the aircraft.

\begin{tabular}{lcc}
\hline Number & $\begin{array}{c}\text { Airborne electronic } \\
\text { countermeasure equipment }\end{array}$ & $S_{15}$ \\
\hline 1 & $\begin{array}{c}\text { Omnidirectional radar warning } \\
\text { equipment }\end{array}$ & 1.05 \\
2 & Ibid + passive jamming dispensing \\
device & 1.10 \\
4 & $\begin{array}{c}\text { Ibid + active infrared and } \\
\text { electromagnetic jammer }\end{array}$ & 1.15 \\
\hline $\begin{array}{c}\text { Ibid + missile approach warning } \\
\text { device }\end{array}$ & 1.20 \\
\hline
\end{tabular}

TABLE 2: Aircraft vulnerability subindexes and definition.

\begin{tabular}{lcc}
\hline Subindexes & Sign & Definition \\
\hline Ratio of fatal components & $S_{21}$ & $S_{21}=\frac{N_{F}}{N}$ \\
Redundant ratio of fatal components & $S_{22}$ & $S_{22}=\frac{N_{R}}{N_{F}}$ \\
Shelter ratio of fatal components & $S_{23}$ & $S_{23}=\sum_{i=1}^{N_{F}} \frac{N_{S i}}{N_{F}}$ \\
Average killing probability & $S_{24}$ & $S_{24}=\sum_{i=1}^{N_{F}} \frac{P_{K i}}{N_{F}}$ \\
Average safety factor & & $S_{25}=\sum_{i=1}^{N_{F}}\left(\frac{n_{D i}}{n_{D i, \max }}\right)$ \\
\hline
\end{tabular}

missiles. However, for electronic countermeasure capability, we can only give a fuzzy value. Electronic countermeasure character can be denoted by the sign $S_{15}$ with dimensionless unit. A typical value can be read in Table 1 .

2.2. Aircraft Vulnerability Subindexes. Aircraft vulnerability refers to the inability of an aircraft to withstand the manmade hostile environment, which lies on ratio of fatal components, redundant ratio of fatal components, shelter ratio of fatal components, average killing probability, and average safety factor. These factors can be defined as is shown in Table 2, where $N_{F}$ is the numbers of fatal components, $N$ is the numbers of whole components, and $N_{S}$ is the numbers of redundant fatal components. $N_{S i}$ is the redundant degree of the $i$ th fatal component. $P_{K i}$ is the killing probability of the $i$ th component while the aircraft is hit. $n_{D i}$ and $n_{D i \text { max }}$ are the designed overload and maximum overload of the $i$ th fatal component, respectively.

Although there are clear equations for the calculation of aircraft vulnerability subindexes, the value of every parameter is comparatively subjective for different definition such as "fatal" and different granularity analysis.

\section{A Multiattribute Intelligent Grey Target Decision Model}

Here we introduce a multiattribute intelligent grey target decision model proposed by Liu et al. [10]. This model takes 
the situation of the shoot and miss of the bull's eye of the objective's effect value and vector based on four kinds of uniform effect measures.

3.1. Problem Description. Assume that $A=\left\{a_{1}, a_{2}, \ldots, a_{n}\right\}$ is the event set, $B=\left\{b_{1}, b_{2}, \ldots, b_{m}\right\}$ the countermeasure set, and $S=\left\{s_{i j}=\left(a_{i}, b_{j}\right) \mid a_{i} \in A, b_{j} \in B\right\}$ the decision set. $\mu_{i j}^{(k)}$ is the effect value of $s_{i j}$ in objective $k$, referring to the similar level or the removed level between the sample and the critical sample.

Assume that $d_{1}^{(k)}$ and $d_{2}^{(k)}$ are the upper and lower critical value $s_{i j}$ in objective $k$. Then $S^{1}=\left\{r \mid d_{1}^{(k)} \leq r \leq d_{2}^{(k)}\right\}$ is the one-dimension grey target and $\mu_{i j}^{(k)} \in\left[d_{1}^{(k)}, d_{2}^{(k)}\right]$ is the pleased effect in objective $k$. Multidimensional grey target can be discussed in the same way. Details are shown in [10].

3.2. Uniform Effect Measures. Considering the decision objectives with different meaning, different dimension, and/or different nature, the effect value of the objective should be transferred to the uniform effect measures.

Assume that $u_{i 0 j 0}^{k}$ is the critical value of objective $k$, and then the grey objective decision of $k$ is designed as follows:

$$
u_{i j}^{k} \in \begin{cases}{\left[u_{i 0 j 0}^{k}, \max _{i} \max _{j}\left\{u_{i j}^{k}\right\}\right],} & k \in \mathrm{BTO} \\ {\left[\min _{i} \min _{j}\left\{u_{i j}^{k}\right\}, u_{i 0 j 0}^{k}\right],} & k \in \mathrm{CTO} \\ {\left[A-u_{i 0 j 0}^{k}, A+u_{i 0 j 0}^{k},\right],} & k \in \mathrm{MTO},\end{cases}
$$

where $k \in \mathrm{BTO}$ means that objective $k$ belongs to benefit type objective, $k \in$ CTO means that objective $k$ belongs to cost type objective, and $k \in$ MTO means that objective $k$ belongs to moderate type objective (same as what follows). And $A$ is the moderate value of the moderate type objective.

For the decision objective of benefit type and cost type, the effect measures $r_{i j}^{k}$ can be shown as

$$
r_{i j}^{k}=\left\{\begin{array}{cl}
\frac{u_{i j}^{k}-u_{i 0 j 0}^{k}}{\max _{i} \max _{j}\left\{u_{i j}^{k}\right\}-u_{i 0 j 0}^{k}}, & k \in \mathrm{BTO} \\
\frac{u_{i 0 j 0}^{k}-u_{i j}^{k}}{u_{i 0 j 0}^{k}-\min _{i} \min _{j}\left\{u_{i j}^{k}\right\}}, & k \in \mathrm{CTO} .
\end{array}\right.
$$

For the decision objective of moderate type, the effect measure can be divided as the upper effect measure and the lower effect measure according to the scale of effect measure $r_{i j}^{k}$, which can be shown as follows:

$$
r_{i j}^{k}= \begin{cases}\frac{u_{i j}^{k}-A+u_{i 0 j 0}^{k}}{u_{i 0 j 0}^{k}}, & u_{i j}^{k} \in\left[A-u_{i 0 j 0}^{k}, A\right], \text { lower effect measure } \\ \frac{A+u_{i 0 j 0}^{k}-u_{i j}^{k}}{u_{i 0 j 0}^{k}}, & u_{i j}^{k} \in\left[A, A+u_{i 0 j 0}^{k}\right], \text { upper effect measure. }\end{cases}
$$

The effect measure of the decision objective of benefit type reflects the similar level between the sample and the biggest sample as well as the removed level between the sample and the critical sample. The effect measure of the decision objective of cost type reflects the similar level between the sample and the smallest sample as well as the removed level between the sample and the critical sample. The lower effect measure of the decision objective of moderate type reflects the level between the sample less than moderate value $A$ and the lower critical sample. The upper effect measure of the decision objective of moderate type reflects the level between the sample greater than moderate value $A$ and the upper critical sample.

The decision objective of benefit type is a type of objective with an expectance the bigger the better or the more the better. The decision objective of cost type is a type of objective with an expectance the smaller the better or the less the better. The decision objective of moderate type is a type of objective with an expectance neither too big nor too small or neither too many nor too few.
The miss of the bull's eye under different conditions can be shown as

$$
\begin{gathered}
u_{i j}^{k}<u_{i 0 j 0}^{k} \quad k \in \mathrm{BTO}, \\
u_{i j}^{k}>u_{i 0 j 0}^{k} \quad k \in \mathrm{CTO}, \\
u_{i j}^{k}<A-u_{i 0 j 0}^{k} \\
\text { or } u_{i j}^{k}>A+u_{i 0 j 0}^{k}
\end{gathered}
$$

$k \in$ MTO.

The effect measure $r_{i j}^{k}$ is satisfied with the following requirements.

(1) $r_{i j}^{k}$ is dimensionless; (2) $r_{i j}^{k}$ is a uniform variable, namely, $r_{i j}^{k} \in[-1,1]$. (3) The greater the $r_{i j}^{k}$, the more ideal the effect. 
Thus in order to satisfy the standardization, the selection of critical value $u_{i 0 j 0}^{k}$ usually satisfies the following:

$$
\begin{aligned}
& u_{i j}^{k} \geq-\max _{i} \max _{j}\left\{u_{i j}^{k}\right\}+2 * u_{i 0 j 0}^{k}, \quad k \in \mathrm{BTO}, \\
& u_{i j}^{k} \leq-\min _{i} \min _{j}\left\{u_{i j}^{k}\right\}+2 * u_{i 0 j 0}^{k}, \quad k \in \mathrm{CTO}, \\
& u_{i j}^{k} \geq A-2 * u_{i 0 j 0}^{k} \\
& \text { or } u_{i j}^{k} \leq A+2 * u_{i 0 j 0}^{k},
\end{aligned}
$$

\section{$k \in$ MTO.}

Assume that $\omega_{k}$ is the decision weight of objective $k$ and $\sum_{k=1}^{s} \omega_{k}=1$. Thus $r_{i j}=\sum_{k=1}^{s} \omega_{k} * r_{i j}^{k}$ will be the synthetic effect measures of decision approach $s_{i j}$ and $R=\left\{r_{i j}\right\}$ will be the matrix of synthetic effect measures of decision set $S$.

The synthetic effect measure $r_{i j}$ is satisfied with the following requirements.

(1) $r_{i j}$ is dimensionless; (2) $r_{i j}$ is a uniform variable, namely, $r_{i j} \in[-1,1]$. (3) The greater the $r_{i j}$, the more ideal the effect.

While $r_{i j} \in[-1,0]$ means the miss of the bull's eye and $r_{i j} \in[0,1]$ means the hit of the bull's eye, through the comparison of the value of $r_{i j}$, we can judge the performance of $a_{i 0}, b_{j 0}$, and $s_{i 0 j 0}$ according to the definition shown as follows.

Definition 1. $b_{j 0}$ is the best decision of event $a_{i}$ if $\max _{1 \leq j \leq m}\left\{r_{i j}\right\}=r_{i j 0} ; a_{i 0}$ is the best event corresponding with the decision $b_{j 0}$ if $\max _{1 \leq i \leq n}\left\{r_{i j}\right\}=r_{i 0 j}$; $s_{i 0 j 0}$ is the best decision approach if $\max _{1 \leq i \leq n} \max _{1 \leq j \leq m}\left\{r_{i j}\right\}=r_{i 0 j 0}$.

3.3. Algorithm Steps. Algorithm steps of the multiattribute intelligent grey target decision model are as follows.

Step 1. Form the decision set $S=\left\{s_{i j}=\left(a_{i}, b_{j}\right) \mid a_{i} \in A, b_{j} \in\right.$ $B\}$.

Step 2. Confirm the decision objective $k=1,2, \ldots, s$.

Step 3. Confirm the decision weight of objective $k \omega_{k}(k=$ $1,2, \ldots, s)$.

Step 4. Form the matrix $U^{k}=\left\{u_{i j}^{k}\right\}$ of effect measures of decision set $S$.

Step 5. Set the critical value of the objective.

Step 6. Obtain the uniform matrix $R^{k}=\left\{r_{i j}^{k}\right\}$ of effect measures.

Step 7. Obtain the uniform matrix $R=\left\{r_{i j}\right\}$ of synthetic effect measures.

Step 8. Confirm the best decision $b_{j 0}$ or the best decision approach $s_{i 0 j 0}$ according to the definitions.
TABLE 3: Exponential scale the golden section.

\begin{tabular}{lc}
\hline Exponential scale & One factor compared to another \\
\hline 1.000 & Equally important \\
1.618 & Slightly more important \\
2.618 & More important \\
4.236 & Greatly more important \\
6.854 & Absolutely more important \\
Reciprocal & Reversed scale \\
\hline
\end{tabular}

\section{Combination Weighting Method}

In Step 3, the decision weight of objective $k \omega_{k}$ is needed. In this paper, according to requirements of the evaluation of aircraft combatant survivability, the evaluation system consists of various criteria with unequal importance, which has a biggish influence on the evaluation. So a rational weighting is necessary. In order to elicit weights, a combination weighting method is brought up based on a modified AHP (analytic hierarchy process) method and the entropy method, offering the subjective weight and the objective weight, respectively.

4.1. Analytic Hierarchy Process Based on Exponential Scale. The AHP is a proven decision-making tool integrating the quantitative analysis and qualitative analysis together, considering the quantitative weight and qualitative weight, into the decision-making process. The AHP has been used widely in a far-ranging field, especially in solving complex decision-making problems with numerous criteria. The AHP establishes a hierarchical structure showing the relationship of the target, criteria, and index, using the decision matrix. The AHP can be broken into five steps as building a hierarchy, making comparisons, calculating weights, checking consistency, and producing the result. The detailed steps are shown in [11].

Dr. Saaty developed a 9-point scale in the pairwise comparisons, which states whether one factor compared to another is important or not. Assign value of 1 to equally important and values of 3,5, 7, and 9 to slightly more important, more important, greatly more important, and absolutely more important. Values of 2, 4, 6, and 8 are reserved for intermediate values. The 9-point scale gives the difference of importance, but the ratio of importance in the pairwise comparisons is what we need according to the AHP. So the AHP based on exponential scale is built. Introduce the ratio of importance $\alpha$ into the comparison as the exponential scale. Replace the values of $1,3,5,7$, and 9 with the values of $1, \alpha, \alpha^{2}, \alpha^{3}$, and $\alpha^{4}$. Assume $\alpha$ meets the rule of ladder by leaps, namely, $a^{k}=a^{k-1}+a^{k-2}$, where $k \in\{2,3,4,5\}$. It turns out to be that $a=1.618$, which satisfied the golden section of importance. The reciprocal scale is achieved when comparing the factors in the opposite direction; that is, if $A$ is more important than $B$ (2.618), $B$ could be said to be less important than $A(1 / 2.618)$. The exponential scale is shown in Table 3.

For the given matrix $M$ through pairwise comparisons, the vector of weights $\omega^{*}$ is the normalized eigenvector of the matrix associated with the largest eigenvalue, $\lambda_{\text {max }}$, using 
TABLE 4: Susceptibility subindexes of different aircraft.

\begin{tabular}{lccccccccccc}
\hline Aircraft & $S_{11}$ & $S_{12}$ & $L$ & $H$ & $W$ & $S_{13}$ & $n_{y \text { max }}$ & $n_{\text {cir }}$ & SEP & $S_{14}$ & $S_{15}$ \\
\hline$A$ & 12.7 & 1672 & 19.43 & 5.63 & 13.05 & 6.985 & 9.0 & 7.5 & 265 & 25.33 \\
$B$ & 5.8 & 1503 & 14.36 & 5.20 & 9.13 & 5.460 & 9.0 & 8.6 & 238 & 25.53 & 1.15 \\
$C$ & 4.9 & 1672 & 15.04 & 5.09 & 10.01 & 5.677 & 9.0 & 7.5 & 290 & 26.17 & 1.15 \\
$D$ & 5.0 & 1756 & 18.4 & 4.88 & 13.6 & 6.631 & 7.0 & 6.0 & 245 & 21.17 & 1.20 \\
$E$ & 0.1 & 1922 & 19.05 & 5.39 & 13.56 & 6.927 & 9.0 & 9.0 & 330 & 29.00 & 1.20 \\
\hline
\end{tabular}

the equation $M \omega^{*}=\lambda_{\max } \omega^{*}$. Thus after calculating and checking of consistency in the matrix, the result is produced.

4.2. Entropy Weight Method. Entropy, according to Shannon's information theory, reflects the degree of disorder of information [12]. In the information system, the smaller the entropy value, the greater the degree of order, and the greater the amount of information. The entropy weight method is an effective method calculating the objective weight, just correlating with the data distribution of the evaluation matrix, without relying on the subjective preference of decisionmaker.

Suppose that there is a matrix $M=\left[m_{i j}\right]_{n \times m}(i=$ $1,2, \ldots, n ; j=1,2, \ldots, m)$ with evaluating indexes counted $m$ and evaluating objects counted $n$. Matrix $P=\left[p_{i j}\right]_{n \times m}$ is the normalized matrix of $M$. So the entropy of the $j$ th index is defined as

$$
E_{j}=-\frac{1}{\log n} \sum_{i=1}^{n} p_{i j} \log p_{i j}
$$

Then the entropy weight of $j$ th index is defined as

$$
\omega_{j}^{\prime}=\frac{\left(1-E_{j}\right)}{\left(m-\sum_{j=1}^{m} E_{j}\right)} .
$$

4.3. Algorithm Steps. The AHP based on exponential scale and entropy weight method are both available in processing the weight; however they have their own advantages and disadvantages. The combination weighting method can evade the disadvantages. The effective combination of subjective weight and objective weight reconciles the expert's preference for the index and the decrease of fuzzy random color, thus producing an advantage of both weighting methods. So the combination weighting method produces a scientific and rational weight.

The combination weight can be defined as

$$
\omega_{j}=\lambda \omega_{j}^{*}+(1-\lambda) \omega_{j}^{\prime}
$$

where $\omega_{j}$ is the combination weight of the $j$ th index; $\omega_{j}^{*}$ is the subjective weight given by the AHP based on exponential scale; $\omega_{j}^{\prime}$ is the objective weight given by the entropy weight method; $\lambda$ is the share of subjective weight in the combination weight.

\section{Evaluation of Aircraft Survivability}

5.1. Data Collection and Pretreatment. Through literature review, data of five aircraft is acquired in susceptibility subindexes, which is shown in Table 4.

But for the vulnerability subindexes, as can be seen ever, which are comparatively subjective, we cannot give a precise value. The vague set is an effective way to solve this problem [13]. For the restriction of the length of this paper, the details will not be discussed here. We know that linguistic variables are available in certainty. Seven-grade linguistic variables $S=\{\mathrm{VG}, \mathrm{G}, \mathrm{FG}, \mathrm{M}, \mathrm{FP}, \mathrm{P}, \mathrm{VP}\}$ presented in vague values are shown in Table 5.

However, with vague value and precise value in one evaluation, it is hard to handle. Here we can transfer the useful information of vague value into a precise value through a score function. We know that a vague value, such as $[0.4,0.7]$, can be interpreted as "the vote for a resolution is 4 in favor, 3 against, and 3 abstentions [13].” Score function can give a certain value of the vague set correlated with a certain value of the fuzzy set, through the undefined and unascertained information of the vague set.

A proved score function contrived by Guo et al. [14] is given as

$$
S= \begin{cases}t_{A}\left(x_{i}\right), & t_{A}\left(x_{i}\right)+f_{A}\left(x_{i}\right)=1 \\ t_{A}\left(x_{i}\right)+\frac{\pi_{A}\left(x_{i}\right)}{2}+\left(t_{A}\left(x_{i}\right)-f_{A}\left(x_{i}\right)\right) \frac{\pi_{A}\left(x_{i}\right)}{2} & 0<t_{A}\left(x_{i}\right)+f_{A}\left(x_{i}\right)<1 \\ -1, & t_{A}\left(x_{i}\right)+f_{A}\left(x_{i}\right)=0,\end{cases}
$$

where $t_{A}\left(x_{i}\right)+f_{A}\left(x_{i}\right)=1$ means that $\pi_{A}\left(x_{i}\right)=0$; that is, the information is absolutely confirmed, so $S=t_{A}(x)$. While
$t_{A}\left(x_{i}\right)+f_{A}\left(x_{i}\right)=0$ means that $\pi_{A}\left(x_{i}\right)=1$, that is, the information is absolutely unascertained, so $S=-1$. 
TABLE 5: Seven-grade linguistic variables of vague value.

\begin{tabular}{lcc}
\hline Grade & Typical vague values & Abstention \\
\hline Very good (VG) & {$[1,1]$} & 0 \\
Good (G) & {$[0.8,0.9]$} & 0.1 \\
Fairly good (FG) & {$[0.6,0.8]$} & 0.2 \\
Medium (M) & {$[0.5,0.5]$} & 0 \\
Fairly poor (FP) & {$[0.2,0.4]$} & 0.2 \\
Poor (P) & {$[0.1,0.2]$} & 0.1 \\
Very poor (VP) & {$[0,0]$} & 0 \\
\hline
\end{tabular}

TABLE 6: Vulnerability subindexes of different aircraft.

\begin{tabular}{|c|c|c|c|c|c|}
\hline Subindexes & $S_{21}$ & $S_{22}$ & $S_{23}$ & $S_{24}$ & $S_{25}$ \\
\hline$A$ & {$[0.2,0.4]$} & {$[0.8,0.9]$} & {$[0.8,0.9]$} & {$[0.2,0.4]$} & {$[0.6,0.8]$} \\
\hline$B$ & {$[0.5,0.5]$} & {$[0.6,0.8]$} & {$[0.6,0.8]$} & {$[0.5,0.5]$} & {$[0.5,0.5]$} \\
\hline C & {$[0.2,0.4]$} & {$[0.6,0.8]$} & {$[0.6,0.8]$} & {$[0.2,0.4]$} & {$[0.5,0.5]$} \\
\hline$D$ & {$[0.1,0.2]$} & {$[1,1]$} & {$[0.6,0.8]$} & {$[0.1,0.2]$} & {$[0.8,0.9]$} \\
\hline$E$ & {$[0.1,0.2]$} & {$[0.8,0.9]$} & {$[0.8,0.9]$} & {$[0.1,0.2]$} & {$[0.6,0.8]$} \\
\hline
\end{tabular}

Through the seven-grade linguistic variables of vague value, we give vulnerability subindexes of different aircraft the value shown in Table 6 .

Thus the subindexes can be normalized as is shown in Table 7. For vulnerability subindexes, a certain value should be given by score function and then the vector normalization method is used. The type of index is also given. Here for the subindexes, there are only too types, where "+" represents benefit type and "-" represents cost type.

5.2. Calculation of Weight. According to AHP based on exponential scale, the subjective weight can be calculated through the introduced five steps. Pairwise comparisons are quite important. Each of the subfactors will need to be compared to each other. There are two approaches generally available. The first is comparing the factors of susceptibility and vulnerability, followed by comparing each subfactor under each factor separately. The other approach is comparing each subfactor to every other one directly, which involves more comparisons. This approach would be difficult and lead to an uncertain result. For instance, trying to compare radar signal character to ratio of fatal components would be difficult, as they are so dissimilar. So the first approach will be used.
Three matrixes of pairwise comparisons given by experts are established. Matrix $P$ is the comparison of susceptibility index and vulnerability index. Define susceptibility index as more important than vulnerability index, thus producing the two by two matrix. $P_{1}$ and $P_{2}$ are the comparison of subindexes of susceptibility and vulnerability, respectively:

$$
\begin{aligned}
P & =\left[\begin{array}{cc}
1.000 & 2.618 \\
\frac{1}{2.618} & 1.000
\end{array}\right], \\
P_{1} & =\left[\begin{array}{ccccc}
1.000 & 1.618 & 4.236 & 2.618 & 1.618 \\
\frac{1}{1.618} & 1.000 & 2.618 & 1.618 & 1.618 \\
\frac{1}{4.236} & \frac{1}{2.618} & 1.000 & \frac{1}{1.618} & \frac{1}{1.618} \\
\frac{1}{2.618} & \frac{1}{1.618} & 1.618 & 1.000 & 1.000 \\
\frac{1}{1.618} & \frac{1}{1.618} & 1.618 & 1.000 & 1.000
\end{array}\right], \\
P_{2} & =\left[\begin{array}{ccccc}
1.000 & 1.618 & 1.618 & 2.618 & 4.236 \\
\frac{1}{1.618} & 1.000 & 1.000 & 1.618 & 2.618 \\
\frac{1}{1.618} & 1.000 & 1.000 & 1.618 & 1.618 \\
\frac{1}{2.618} & \frac{1}{1.618} & \frac{1}{1.618} & 1.000 & 2.618 \\
\frac{1}{4.236} & \frac{1}{2.618} & \frac{1}{1.618} & \frac{1}{2.618} & 1.000
\end{array}\right] .
\end{aligned}
$$

The next steps are calculating weights and checking consistency. The calculation of $P$ is $\omega_{I}^{*}=(0.7236,0.2764)$, and the calculation of $P_{1}$ and $P_{2}$ is as follows:

$$
\begin{aligned}
& \omega_{1}^{*}=(0.3553,0.2399,0.0916,0.1483,0.1649), \\
& \omega_{2}^{*}=(0.3496,0.2160,0.1993,0.1502,0.0848) .
\end{aligned}
$$

Reference [11] gives the method of checking consistency, using the equation $\mathrm{CR}=\mathrm{CI} / \mathrm{RI}$, where $\mathrm{CI}=\left(\lambda_{\max }-n\right) /(n-$ $1), \mathrm{RI}$ is the random consistency index, $\lambda_{\max }$ is maximum eigenvalue of the matrix, and $n$ is the number of factors. The consistency of matrixes $P_{1}$ and $P_{2}$ is 0.0063 and 0.0168 , respectively, which is less than 10 percent; that is, the matrix is consistent enough and can be used for calculating results.

So the subjective weight of all subindexes is

$$
\omega^{*}=(0.2571,0.1736,0.0663,0.1073,0.1193,0.0966,0.0597,0.0551,0.0415,0.0234) .
$$

The entropy weight can be calculated using the entropy weight method as

$$
\omega^{\prime}=(0.1601,0.0810,0.1045,0.0815,0.0804,0.1210,0.0820,0.0812,0.1210,0.0873) .
$$


TABLE 7: The normalization of the subindexes.

\begin{tabular}{lcccccccccc}
\hline Subindexes & $S_{11}$ & $S_{12}$ & $S_{13}$ & $S_{14}$ & $S_{15}$ & $S_{21}$ & $S_{22}$ & $S_{23}$ & $S_{24}$ & $S_{25}$ \\
\hline Type & - & - & - & + & + & - & + & + & - \\
$A$ & 0.813 & 0.437 & 0.491 & 0.443 & 0.447 & 0.405 & 0.462 & 0.494 & 0.405 & 0.480 \\
$B$ & 0.371 & 0.393 & 0.383 & 0.447 & 0.408 & 0.779 & 0.387 & 0.413 & 0.779 & 0.324 \\
$C$ & 0.314 & 0.437 & 0.399 & 0.458 & 0.447 & 0.405 & 0.387 & 0.413 & 0.405 & 0.324 \\
$D$ & 0.320 & 0.459 & 0.466 & 0.370 & 0.466 & 0.179 & 0.523 & 0.413 & 0.179 & 0.574 \\
$E$ & 0.006 & 0.503 & 0.486 & 0.507 & 0.466 & 0.179 & 0.462 & 0.494 & 0.179 & 0.480 \\
\hline
\end{tabular}

Then the combination weight $\omega$ can be calculated in (11), where $\lambda=0.5$ as follows:

$$
\omega=(0.2086,0.1273,0.0854,0.0944,0.09985,0.1088,0.07085,0.06815,0.08125,0.05535) .
$$

5.3. Multiattribute Intelligent Grey Target Decision. Here we give an evaluation of aircraft survivability. The evaluation of aircraft survivability is the event $a_{1}$, so $A=\left\{a_{1}\right\}$. The aircraft $A, B, C, D$, and $E$ are the countermeasures $b_{1}, b_{2}, b_{3}, b_{4}, b_{5}$, so $B=\left\{b_{1}, b_{2}, b_{3}, b_{4}\right.$, and $\left.b_{5}\right\}$. Thus we can form the decision set $S=\left\{s_{11}, s_{12}, s_{13}, s_{14}, s_{15}\right\}$.

As is shown above, the susceptibility subindexes and vulnerability subindexes of different aircraft are chosen as the decision objective $k=1,2, \ldots, 10$. For the decision objective of benefit type, the critical value is $u_{i 0 j 0}^{k}=1922, k=$ $1,2,3,6,9$. For the decision objective of cost type, the critical value is $u_{i 0 j 0}^{k}=0.5, k=4,5,7,8,10$. The decision weight of objective $k$ has been given above. The matrix $U^{k}=\left\{u_{i j}^{k}\right\}$ of effect measures of decision set $S$ is formed according to the collected data. Thus the uniform effect measures matrix $R^{k}=$ $\left\{r_{i j}^{k}\right\}$ is obtained with the defined effect measure for benefit type objective and effect measure for cost type objective, shown as follows:

$$
R^{k}=\left[\begin{array}{lllll}
0.0000 & 0.5476 & 0.6190 & 0.6111 & 1.0000 \\
0.5967 & 1.0000 & 0.5967 & 0.3962 & 0.0000 \\
0.0000 & 1.0000 & 0.8577 & 0.2321 & 0.0380 \\
0.5313 & 0.5568 & 0.6386 & 0.0000 & 1.0000 \\
0.6667 & 0.0000 & 0.6667 & 1.0000 & 1.0000 \\
0.6234 & 0.0000 & 0.6234 & 1.0000 & 1.0000 \\
0.5577 & 0.0000 & 0.0000 & 1.0000 & 0.5577 \\
1.0000 & 0.0000 & 0.0000 & 0.0000 & 1.0000 \\
0.6234 & 0.0000 & 0.6234 & 1.0000 & 1.0000 \\
0.6234 & 0.0000 & 0.0000 & 1.0000 & 0.6234
\end{array}\right] .
$$

Through the equation $r_{i j}=\sum_{k=1}^{s} \omega_{k} * r_{i j}^{k}$, the uniform matrix $R=\left\{r_{i j}\right\}$ of synthetic effect measures can be obtained as $R=[0.4533,0.3795,0.5237,0.6138,0.7383]$. Then according to the definitions, the best countermeasure is $b_{5}$; that is, the aircraft $E$ is the best decision and has the best aircraft survivability. Through the ranking of $r_{i j}$, we can give a ranking of aircraft, $E>D>C>A>B$.

A rational weight is essential. An opposite example with equal weight for every subindex is given. The weight is 0.1 . For the restriction of the length of this paper, we just list the result of the uniform matrix of synthetic effect measures, $R=$ $[0.8782,0.7764,0.7527,0.8742,0.8393]$. Here, the aircraft $A$ is the best decision and has the best aircraft survivability. This result is quite different. And the difference of every aircraft is little, which is hard to give a rational evaluation.

With the calculated weight according to AHP based on exponential scale and the entropy weight method, we can just calculate the susceptibility of the aircraft using the same method. According to the algorithm steps, the uniform matrix of synthetic effect measures can be obtained as $R_{S}=[0.8244,0.8136,0.8304,0.8106,0.7580]$. Then according to the definitions, the best countermeasure is $b_{3}$; that is, the aircraft $C$ is the best decision and has the best aircraft susceptibility. Through the ranking of $r_{i j}$, we can give a ranking of aircraft, $C>A>B>D>E$. However, this result is so different with the aircraft survivability. Some reasons can be listed. Firstly, aircraft susceptibility and aircraft survivability are different in definitions and evaluation subindexes. Then, although turbine inlet temperature is a good evaluation index for the infrared signal character, different from RCS, turbine inlet temperature is not enough especially for the new generation aircraft. Turbine inlet temperature is not the sole influence factor of infrared signal character. Infrared suppressing functions such as stealth materials and thermal isolation have no influence on turbine inlet temperature but have great influence on infrared signal character. This is the future work to establish a more rational index system.

Then we calculate the vulnerability of the aircraft. The combination weight has been given in the former section. The uniform matrix of synthetic effect measures is obtained as $R_{V}=[0.7300,0.2279,0.5395,0.9248,0.9183]$. Then the best countermeasure is $b_{4}$; that is, the aircraft $D$ is the best decision 
and has the best aircraft vulnerability. The ranking of aircraft vulnerability is $D>E>A>C>B$.

\section{Conclusions}

With the development of precision guided weapons, aircraft combat survivability has been increasingly important. Multicriteria decision method is a useful method through ranking and selecting a finite number of alternative plans. In this paper, in order to give a rational evaluation of aircraft combat survivability, a new multiattribute intelligent grey target decision model is introduced. Conclusions can be shown as follows:

(1) The aircraft combat survivability evaluation index system is established in hierarchy containing susceptibility index and vulnerability index and their subindexes, which is essential to aircraft survivability.

(2) The multiattribute intelligent grey target decision model is introduced, which not only can be used in the evaluation of aircraft combat survivability, but also can be used in any similar evaluation questions.

(3) Then a combination weighting method is brought up based on a modified AHP (analytic hierarchy process) method and the entropy method, offering the combination weight to the multiattribute intelligent grey target decision model, which can evade the disadvantages. This method produces a scientific and rational weight, improving the accuracy and objectivity of the evaluation, which can be used in any evaluation requiring a rational weight.

(4) In the end, a numerical example containing five aircraft is given, proving that this method is practicable and effective. The result of more numerical calculation can provide more details, to find the most important influencing factors, which can be used in the program design of new aircraft and modified design of the old aircraft.

However, we did not consider the real states in the combat, such as detected, tracked, or hit, as well as the influence of aircraft combat capability. So future work will be carried out on in directions: (1) establish a more rational evaluation system and find more rational subindexes, such as the representation of infrared signal character; (2) consider the real states in the combat, the threats, and aircraft combat capability to give capability of an aircraft to avoid or withstand a man-made hostile environment.

\section{Conflict of Interests}

The authors declare that there is no conflict of interests regarding the publication of this paper.

\section{References}

[1] Military Handbook: Aircraft Survivability. MIL-HDBK-2069, U. S. Department of Defense, 2069.
[2] X. Wang, B.-F. Song, and Y.-F. Hu, "Analytic model for aircraft survivability assessment of a one-on-one engagement," Journal of Aircraft, vol. 46, no. 1, pp. 223-229, 2009.

[3] H. E. Konokman, A. Kayran, and M. Kaya, Analysis of Aircraft Survivability Against Fragmenting Warhead Threat, American Institute of Aeronautics and Astronautics, National Harbor, Md, USA, 2014.

[4] J. Li, W. Yang, Y. Zhang, Y. Pei, Y. Ren, and W. Wang, "Aircraft vulnerability modeling and computation methods based on product structure and CATIA," Chinese Journal of Aeronautics, vol. 26, no. 2, pp. 334-342, 2013.

[5] T. Erlandsson and L. Niklasson, "A five states survivability model for missions with ground-to-air threats," in Modeling and Simulation for Defense Systems and Applications VIII, vol. 8752 of Proceedings of SPIE, pp. 1-7, May 2013.

[6] S. Shi, B.-F. Song, Y. Pei, and T. Cheng, "Assessment method of aircraft susceptibility based on agent theory," Acta Aeronautica et Astronautica Sinica, vol. 35, no. 2, pp. 444-453, 2014 (Chinese).

[7] S. F. Liu and N. M. Xie, Grey Systems Theory and Application, Science Press, Beijing, China, 4th edition, 2008 (Chinese).

[8] S.-H. Ahn, Y.-C. Kim, T.-W. Bae, B.-I. Kim, and K.-H. Kim, "DIRCM jamming effect analysis of spin-scan reticle seeker," in Proceedings of the IEEE 9th Malaysia International Conference on Communications with a Special Workshop on Digital TV Contents (MICC '09), pp. 183-186, Kuala Lumpur, Malaysia, December 2009.

[9] J. Paterson, "Overview of low observable technology and its effects on combat aircraft survivability," Journal of Aircraft, vol. 36, no. 2, pp. 380-388, 1999.

[10] S.-F. Liu, W.-F. Yuan, and K.-Q. Sheng, "Multi-attribute intelligent grey target decision model," Control and Decision, vol. 25, no. 8, pp. 1159-1163, 2010.

[11] M. J. Tabar, Analysis of Decisions Made Using the Analytic Hierarchy Process, Naval Postgraduate School, Monterey, Calif, USA, 2013.

[12] Z. Yi and W. Zhuo-Fu, "Bid evaluation research of construction project based on two-stage entropy weight," in Proceedings of the International Conference on Information Management, Innovation Management and Industrial Engineering (ICIII '09), vol. 2, pp. 133-135, IEEE, Xi'an, China, December 2009.

[13] W.-L. Gau and D. J. Buehrer, "Vague sets," IEEE Transactions on Systems, Man and Cybernetics, vol. 23, no. 2, pp. 610-614, 1993.

[14] R. Guo, J. Guo, Y.-B. Su, and Y.-D. Zhang, "Ranking limitation and improvement strategy of vague sets based on score function," Systems Engineering and Electronics, vol. 36, no. 1, pp. 105110, 2014 (Chinese). 


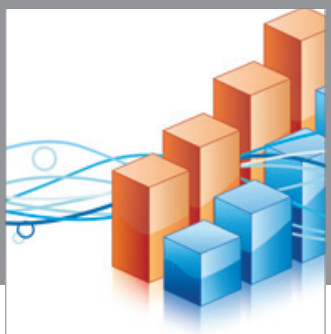

Advances in

Operations Research

vatem alat4

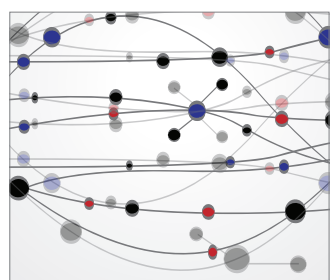

\section{The Scientific} World Journal
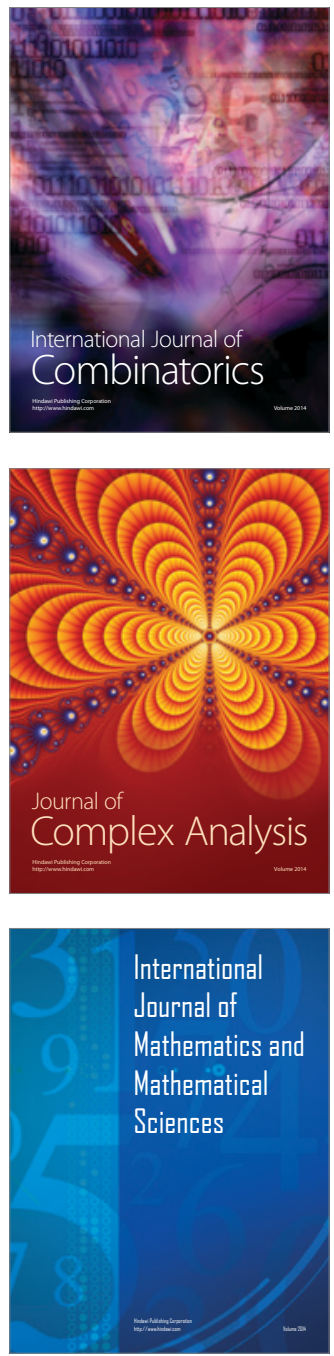
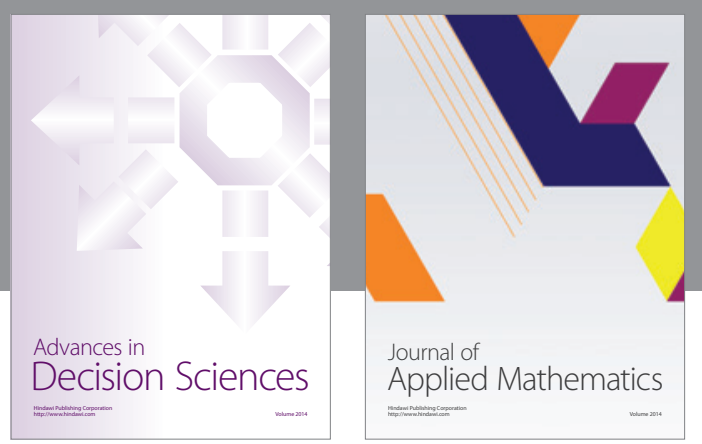

Algebra

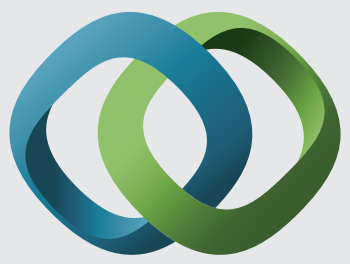

\section{Hindawi}

Submit your manuscripts at

http://www.hindawi.com
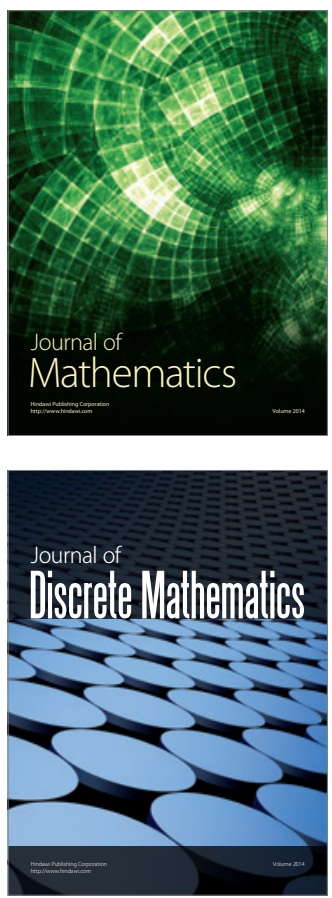

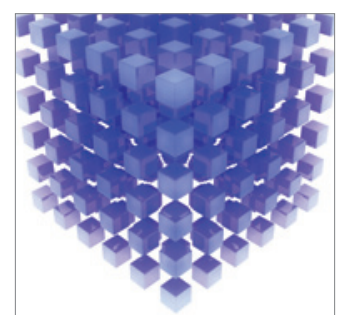

Mathematical Problems in Engineering
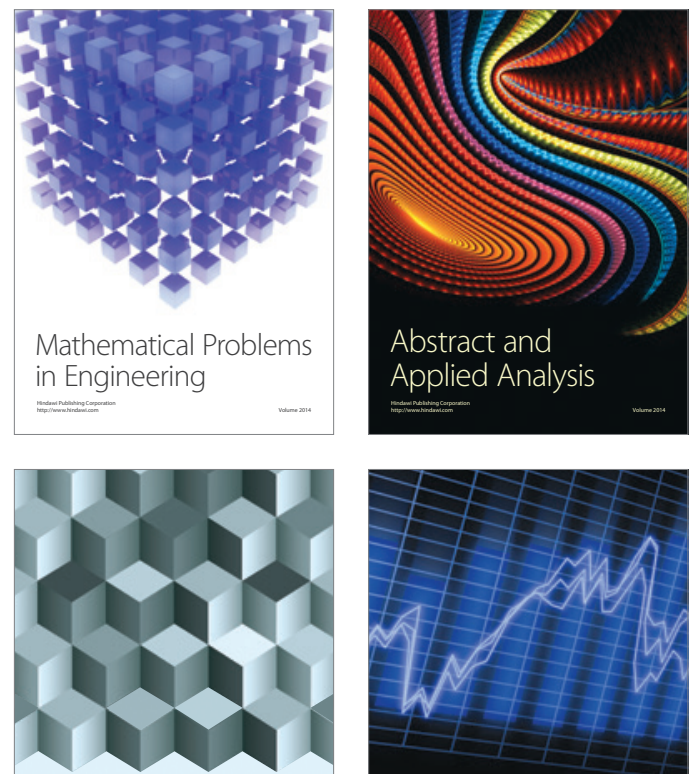

Journal of

Function Spaces

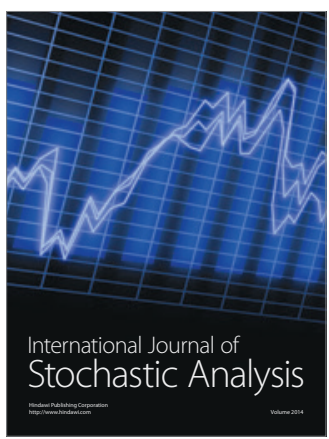

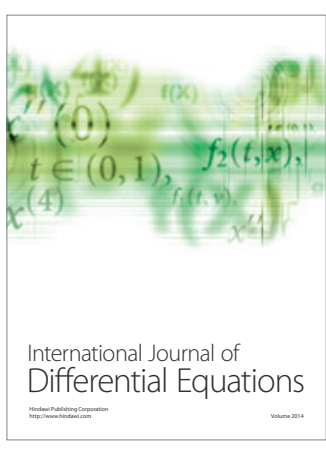
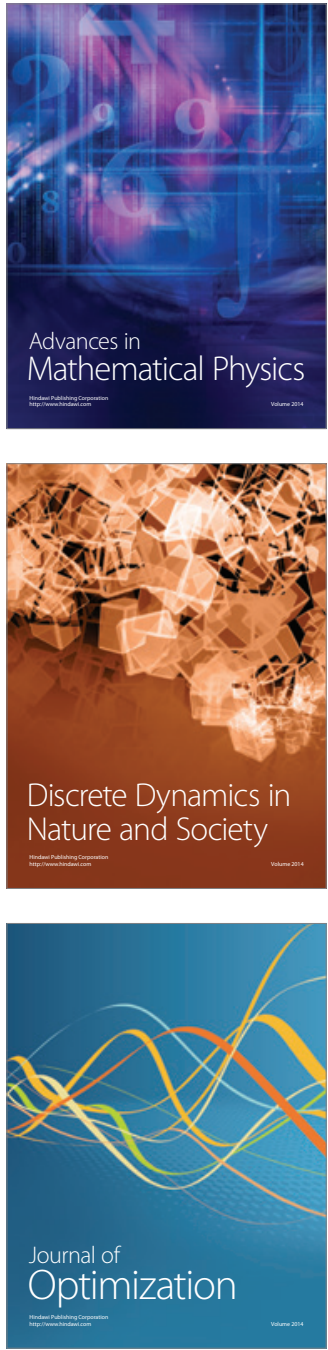